\title{
A fast deisotoping algorithm and its implementation in the MSFragger search engine
}

\author{
Guo Ci Teo ${ }^{1 \#}$, Daniel A. Polasky", Fengchao Yu¹, Alexey I. Nesvizhskii¹,2 \\ ${ }^{1}$ Department of Pathology, University of Michigan, Ann Arbor, Michigan, USA \\ ${ }^{2}$ Department of Computational Medicine and Bioinformatics, University of Michigan, Ann Arbor, \\ Michigan, USA \\ *Correspondence to A.I.N. (nesvi@med.umich.edu) \\ \#contributed equally
}

\section{Table of Contents}

1. Table S1. Accession numbers and details for all datasets searched

2. Table S2. Speed comparison across deisotoping tools

3. Table S3. Deisotoping tool outputs compared for proteomics database search with MSFragger

4. Table S4. Effect of spectrum preprocessing on improvement from deisotoping

5. Table S5. Average PSM precursor mass for several datasets

6. Figure S1. Examples of separating overlapped isotope distributions

7. Figure S2. Example spectrum from Ammar et al.

8. Figure S3. Histogram of number of peaks in MS2 spectra from Ammar et al.

9. Figure S4. Distribution of MS2 peak intensities from Chick and Ammar et al. datasets

10. Supplementary Files. MSFragger and Philosopher parameter files used for all searches and HardKlor and MS2Deisotoper parameters used (zip archive of text files). 
Table S1. Dataset details for all datasets searched. Search name is how the data is referred to in the manuscript. Full sample, preparation, and acquisition details can be found in the corresponding publications for each study.

\begin{tabular}{|c|c|c|c|c|}
\hline Search Name & $\begin{array}{l}\text { Accession } \\
\text { Number }\end{array}$ & Reference & Instrument & Sample \\
\hline Closed & PXD001468 & Chick et al. ${ }^{1}$ & $\begin{array}{c}\text { Thermo Orbitrap } \\
\text { Q Exactive }\end{array}$ & $\begin{array}{l}\text { peptides from HEK293 cell } \\
\text { digest, } 24 \text { fractions }\end{array}$ \\
\hline $\begin{array}{l}\text { Open (same } \\
\text { as QExactive) }\end{array}$ & PXD001468 & Chick et al. ${ }^{1}$ & $\begin{array}{c}\text { Thermo Orbitrap } \\
\text { Q Exactive }\end{array}$ & $\begin{array}{l}\text { peptides from HEK293 cell } \\
\text { digest, } 24 \text { fractions }\end{array}$ \\
\hline Glyco & PXD011533 & Riley et al. ${ }^{2}$ & $\begin{array}{l}\text { Thermo Orbitrap } \\
\text { Fusion Lumos }\end{array}$ & $\begin{array}{l}\text { Mouse brain } \mathrm{N} \text {-glycopeptides } \\
\text { (lectin enriched) analyzed by } \\
\text { HCD-pd-AIETD }\end{array}$ \\
\hline Nonspecific & PXD013662 & $\begin{array}{c}\text { Anapindi et } \\
\text { al. }^{3}\end{array}$ & $\begin{array}{l}\text { Thermo Orbitrap } \\
\text { Fusion }\end{array}$ & $\begin{array}{l}\text { endogenous neuropeptides } \\
\text { extracted from mouse, only } \\
\text { TG (trigeminal ganglia) } \\
\text { samples used here }\end{array}$ \\
\hline Fusion Lumos & PXD004940 & $\begin{array}{c}\text { Espadas et } \\
\text { al. }^{4}\end{array}$ & $\begin{array}{l}\text { Thermo Orbitrap } \\
\text { Fusion Lumos }\end{array}$ & $\begin{array}{l}\text { single-run HeLa cell digest } \\
\text { peptides, only non-enriched } \\
\text { HCD-Oribtrap data used here }\end{array}$ \\
\hline $\begin{array}{l}\text { QExactive } \\
\text { (same as } \\
\text { Open) }\end{array}$ & PXD001468 & Chick et al. ${ }^{1}$ & $\begin{array}{c}\text { Thermo Orbitrap } \\
\text { Q Exactive }\end{array}$ & $\begin{array}{l}\text { peptides from HEK293 cell } \\
\text { digest, } 24 \text { fractions }\end{array}$ \\
\hline timsTOF & PXD010012 & Meier et al. ${ }^{5}$ & $\begin{array}{c}\text { Bruker timsTOF } \\
\text { Pro } \\
\end{array}$ & $\begin{array}{l}\text { single-run DDA-PASEF } \\
\text { acquisition of HeLa cell } \\
\text { digest peptides }\end{array}$ \\
\hline Sciex & PXD005111 & Ammar et al. ${ }^{6}$ & $\begin{array}{c}\text { SciEx tripleTOF } \\
6600+ \\
\end{array}$ & $\begin{array}{l}\text { single-run acquisition of } \\
\text { Jurkat cell digest }\end{array}$ \\
\hline
\end{tabular}


Table S2. Speed comparison across deisotoping tools. Time to deisotope raw files from the Chick et al. data is reported for MSFragger, Hardklor ${ }^{7,8}$, and MS2Deisotoper ${ }^{9}$. MSFragger and Hardklor report deisotoping analysis time only, while MS2Deisotoper only reports total time. All testing was performed on a linux server with 28 cores (56 threads) and 500 GB RAM. As MSFragger is multi-threaded while the other tools are not, we restricted the maximum threads to 8 to simulate typical performance on a desktop computer. Restricted to 8 threads, MSFragger deisotoping was roughly 100 times faster than Hardklor and more than 1,000 times faster than MS2Deisotoper.

\begin{tabular}{|c|c|c|c|c|c|c|}
\hline & & MSFr & agger tim & $(s)$ & & \\
\hline Raw File & $\begin{array}{l}\text { MS2 } \\
\text { scans }\end{array}$ & $\begin{array}{c}56 \\
\text { threads }\end{array}$ & $\begin{array}{c}8 \\
\text { threads }\end{array}$ & $\begin{array}{c}1 \\
\text { thread }\end{array}$ & $\begin{array}{c}\text { Hardklor } \\
\text { time }(s)\end{array}$ & $\begin{array}{c}\text { MS2 } \\
\text { Deisotoper } \\
\text { time }(s)^{*}\end{array}$ \\
\hline b1906_293T_proteinID_01A_QE3_122212 & 41,808 & 0.1 & 0.2 & 1.7 & 24 & 319 \\
\hline b1922_293T_proteinID_02A_QE3_122212 & 46,842 & 0.1 & 0.3 & 2.1 & 26 & 384 \\
\hline b1923_293T_proteinID_03A_QE3_122212 & 42,649 & 0 & 0.2 & 1.2 & 17 & 232 \\
\hline b1924_293T_proteinID_04A_QE3_122212 & 48,007 & 0.1 & 0.2 & 1.8 & 26 & 345 \\
\hline b1925_293T_proteinID_05A_QE3_122212 & 48,751 & 0.1 & 0.2 & 1.5 & 21 & 271 \\
\hline b1926_293T_proteinID_06A_QE3_122212 & 42,791 & 0 & 0.2 & 1.5 & 20 & 265 \\
\hline b1927_293T_proteinID_07A_QE3_122212 & 52,236 & 0.1 & 0.2 & 1.7 & 24 & 336 \\
\hline b1928_293T_proteinID_08A_QE3_122212 & 44,010 & 0 & 0.2 & 1.5 & 21 & 280 \\
\hline b1929_293T_proteinID_09A_QE3_122212 & 58,802 & 0.1 & 0.3 & 2.5 & 34 & 473 \\
\hline b1930_293T_proteinID_10A_QE3_122212 & 45,083 & 0 & 0.2 & 1.4 & 20 & 263 \\
\hline b1931_293T_proteinID_11A_QE3_122212 & 55,320 & 0.1 & 0.2 & 2.3 & 32 & 437 \\
\hline b1932_293T_proteinID_12A_QE3_122212 & 41,265 & 0 & 0.2 & 1.2 & 16 & 234 \\
\hline b1937_293T_proteinID_01B_QE3_122212 & 46,774 & 0 & 0.2 & 1.5 & 21 & 280 \\
\hline b1938_293T_proteinID_02B_QE3_122212 & 45,610 & 0 & 0.2 & 1.3 & 18 & 233 \\
\hline b1939_293T_proteinID_03B_QE3_122212 & 51,243 & 0.1 & 0.2 & 1.9 & 26 & 353 \\
\hline b1940_293T_proteinID_04B_QE3_122212 & 48,937 & 0 & 0.2 & 1.3 & 19 & 267 \\
\hline b1941_293T_proteinID_05B_QE3_122212 & 44,088 & 0 & 0.2 & 1.4 & 21 & 271 \\
\hline b1942_293T_proteinID_06B_QE3_122212 & 54,006 & 0.1 & 0.2 & 1.6 & 24 & 308 \\
\hline b1943_293T_proteinID_07B_QE3_122212 & 39,841 & 0 & 0.1 & 1 & 15 & 193 \\
\hline b1944_293T_proteinID_08B_QE3_122212 & 58,432 & 0.1 & 0.3 & 2 & 29 & 391 \\
\hline b1945_293T_proteinID_09B_QE3_122212 & 17,579 & 0 & 0 & 0.2 & 2 & 43 \\
\hline b1946_293T_proteinID_10B_QE3_122212 & 57,819 & 0.1 & 0.3 & 2.3 & 33 & 462 \\
\hline b1947_293T_proteinID_11B_QE3_122212 & 32,923 & 0 & 0.1 & 0.7 & 9 & 126 \\
\hline b1948_293T_proteinID_12B_QE3_122212 & 55,112 & 0.1 & 0.3 & 2.2 & 31 & 415 \\
\hline Total & $1,119,928$ & 1.2 & 4.9 & 37.8 & 529 & 7178 \\
\hline
\end{tabular}


Table S3. Deisotoping tool outputs compared for proteomics database search with MSFragger. MS2Deisotoper and MSConvert ${ }^{10,11}$ both improved the number of PSMs detected vs no deisotoping, but not as much as MSFragger for both open and closed searches. Hardklor reduced PSMs detected in both searches by a large margin, possibly due to reporting many fewer peaks per spectrum than MSFragger or MS2Deisotoper, even when the minimum score threshold was reduced well below the recommended setting.

\begin{tabular}{|c|c|c|c|c|}
\hline Search Type & Deisotoping method & PSMs & Peptides & Proteins \\
\hline \multirow{4}{*}{$\begin{array}{c}\text { Closed - Chick et } \\
\text { al. }\end{array}$} & none & 568,614 & 129,100 & 9,324 \\
\cline { 2 - 5 } & MSFragger & 572,142 & 129,424 & 9,303 \\
\cline { 2 - 5 } & MS2 Deisotoper & 569,856 & 129,247 & 9,275 \\
\cline { 2 - 5 } & MSConvert & 570,462 & 129,285 & 9,285 \\
\cline { 2 - 5 } & HardKlor & 278,686 & 82,731 & 7,732 \\
\hline \multirow{4}{*}{$\begin{array}{c}\text { Open - Chick et } \\
\text { al. }\end{array}$} & none & 747,152 & 137,542 & 9,489 \\
\cline { 2 - 5 } & MSFragger & 762,883 & 139,543 & 9,524 \\
\cline { 2 - 5 } & MS2 Deisotoper & 751,921 & 138,064 & 9,501 \\
\cline { 2 - 5 } & MSConvert & 752,661 & 138,131 & 9,498 \\
\cline { 2 - 5 } & HardKlor & 302,547 & 83,002 & 7,815 \\
\hline
\end{tabular}


Table S4. Effect of spectrum preprocessing on improvement from deisotoping. Mass calibration and top $\mathrm{N}$ peaks filter had only minor impacts on the degree of improvement with deisotoping compared to standard parameters, whereas increasing the fragment mass tolerance from 10 $\mathrm{ppm}$ to $0.02 \mathrm{Da}$ had a clear impact. Removing all three (calibration, topN filter, and increasing fragment tolerance) resulted in an improvement of $3.1 \%$ with deisotoping.

\begin{tabular}{|c|c|c|c|c|c|c|}
\hline $\begin{array}{l}\text { Search } \\
\text { Type }\end{array}$ & Parameters & Deisotoping? & PSMs & Peptides & Proteins & $\begin{array}{c}\text { PSM } \\
\text { improvement } \\
\text { with } \\
\text { deisotoping }\end{array}$ \\
\hline \multirow{2}{*}{$\begin{array}{l}\text { Closed } \\
\text { Chick et al. }\end{array}$} & \multirow{2}{*}{ standard } & no & 568,614 & 129,100 & 9,324 & \multirow{2}{*}{$0.6 \%$} \\
\hline & & yes & 572,142 & 129,424 & 9,303 & \\
\hline \multirow{2}{*}{$\begin{array}{l}\text { Closed } \\
\text { Chick et al. }\end{array}$} & \multirow{2}{*}{ no mass calibration } & no & 566,901 & 128,852 & 9,310 & \multirow{2}{*}{$0.7 \%$} \\
\hline & & yes & 571,081 & 129,304 & 9,285 & \\
\hline \multirow{2}{*}{$\begin{array}{l}\text { Closed } \\
\text { Chick et al. }\end{array}$} & \multirow{2}{*}{ no topN filter } & no & 568,481 & 129,043 & 9,295 & \multirow{2}{*}{$0.6 \%$} \\
\hline & & yes & 572,049 & 129,314 & 9,264 & \\
\hline \multirow{2}{*}{$\begin{array}{c}\text { Closed } \\
\text { Chick et al. }\end{array}$} & \multirow{2}{*}{$\begin{array}{c}\text { fragment tolerance } \\
0.02 \mathrm{Da} \\
\end{array}$} & no & 555,752 & 126,794 & 9,305 & \multirow{2}{*}{$1.8 \%$} \\
\hline & & yes & 565,823 & 128,215 & 9,316 & \\
\hline \multirow{2}{*}{$\begin{array}{l}\text { Closed } \\
\text { Chick et al. }\end{array}$} & \multirow{2}{*}{$\begin{array}{c}\text { no cal, no topN, and } \\
\text { fragmt tol } 0.02 \mathrm{Da}\end{array}$} & no & 545,249 & 124,717 & 9,243 & \multirow{2}{*}{$3.1 \%$} \\
\hline & & yes & 562,097 & 127,403 & 9,291 & \\
\hline
\end{tabular}

Table S5. Average precursor mass (of PSMs passing FDR) for several datasets. The glycoproteomic and peptidomic searches had much larger precursors on average than standard tryptic digest datasets.

\begin{tabular}{|c|c|c|}
\hline Dataset & Data Type & $\begin{array}{c}\text { Average } \\
\text { precursor } \\
\text { mass (Da) }\end{array}$ \\
\hline Riley et al. & glyco & 2687 \\
\hline Anapindi et al. & peptidome & 2883 \\
\hline Chick et al. & standard & 1585 \\
\hline Espadas et al. & standard & 1510 \\
\hline Meier et al. & standard & 1796 \\
\hline
\end{tabular}



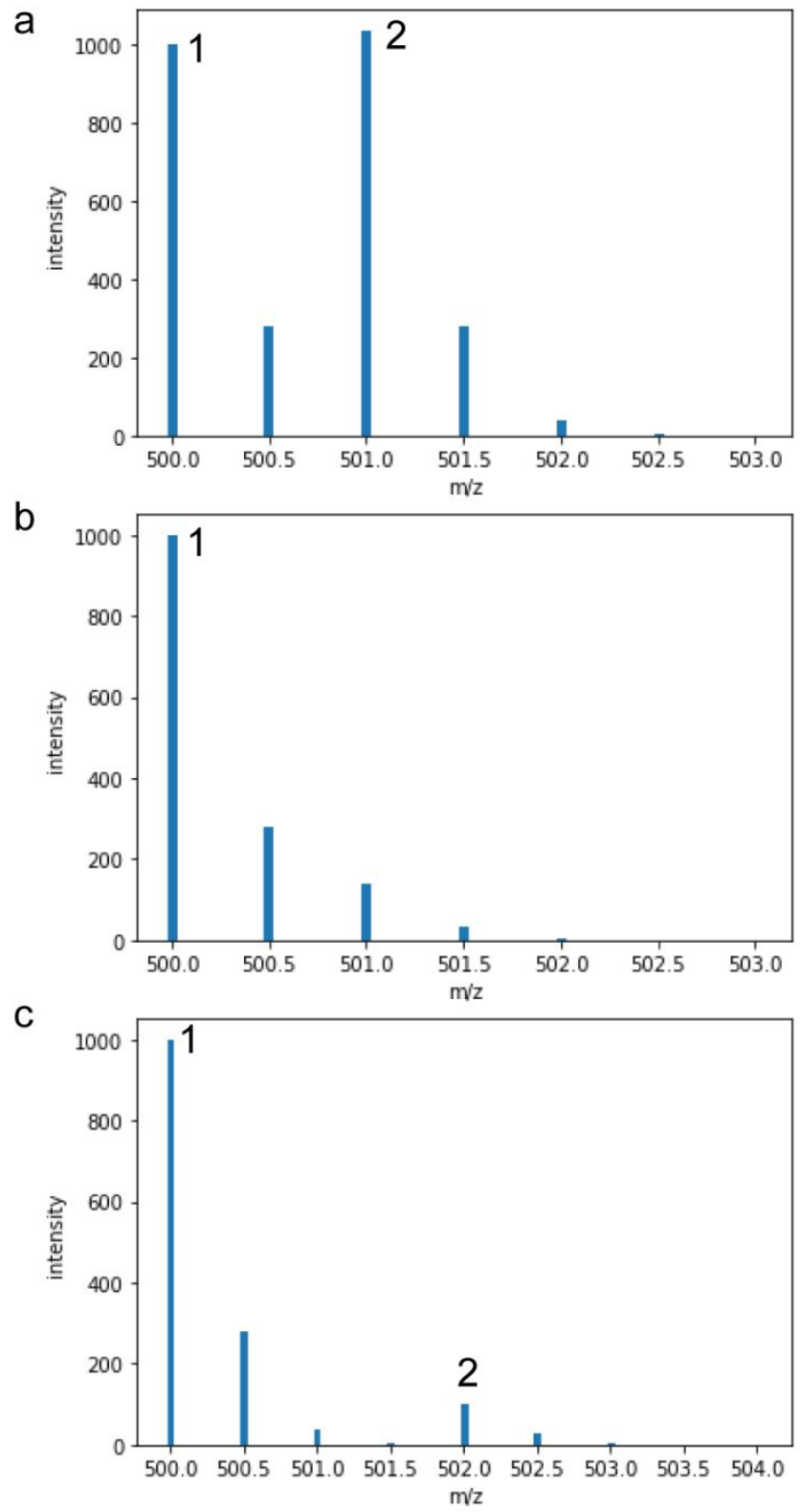

Figure S1. Examples of overlapped isotope distributions. a) Two overlapping clusters, with the second cluster starting from the third peak of the first cluster, and both have the same intensities. In this case, the algorithm separates. b) The same clusters as in (a), but with the intensity of cluster 2 reduced by a factor of 10 , causing the algorithm to fail to separate the clusters. c) The same clusters as in (b), but with the second cluster moved to start at the $5^{\text {th }}$ peak of cluster 1 instead of the $3^{\text {rd }}$, which restores the separate identification of the two clusters. 


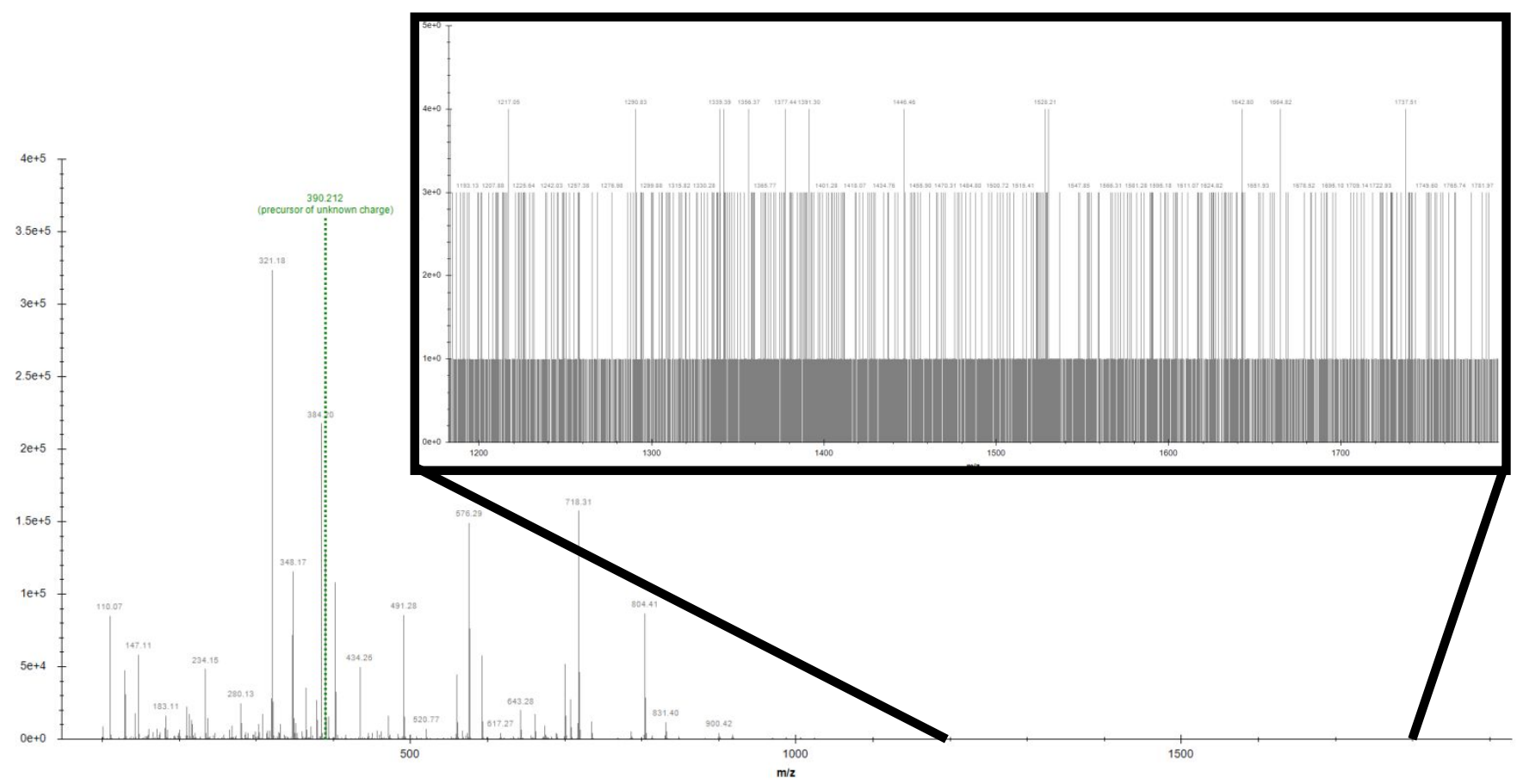

Figure S2. Example spectrum from Ammar et al. data showing zoomed view of hundreds of noise peaks appearing along the baseline. 


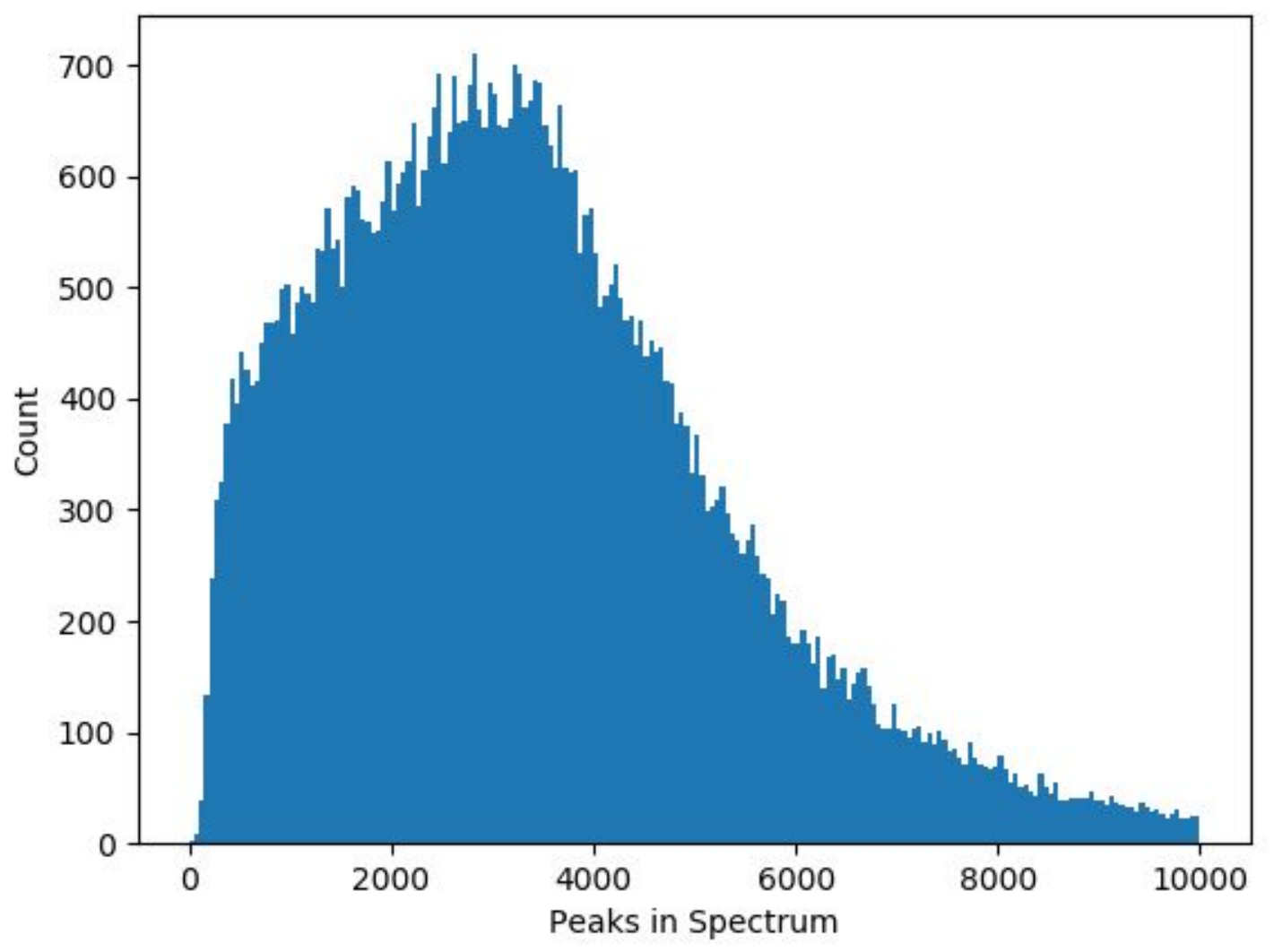

Figure S3. Histogram of number of peaks per MS2 spectrum from Ammar et al. TripleTOF $6600+$ data. The plot is cut off at 10,000 peaks, but the right tail of the distribution continues to a maximum of 97,579 peaks in a single MS2 spectrum. 

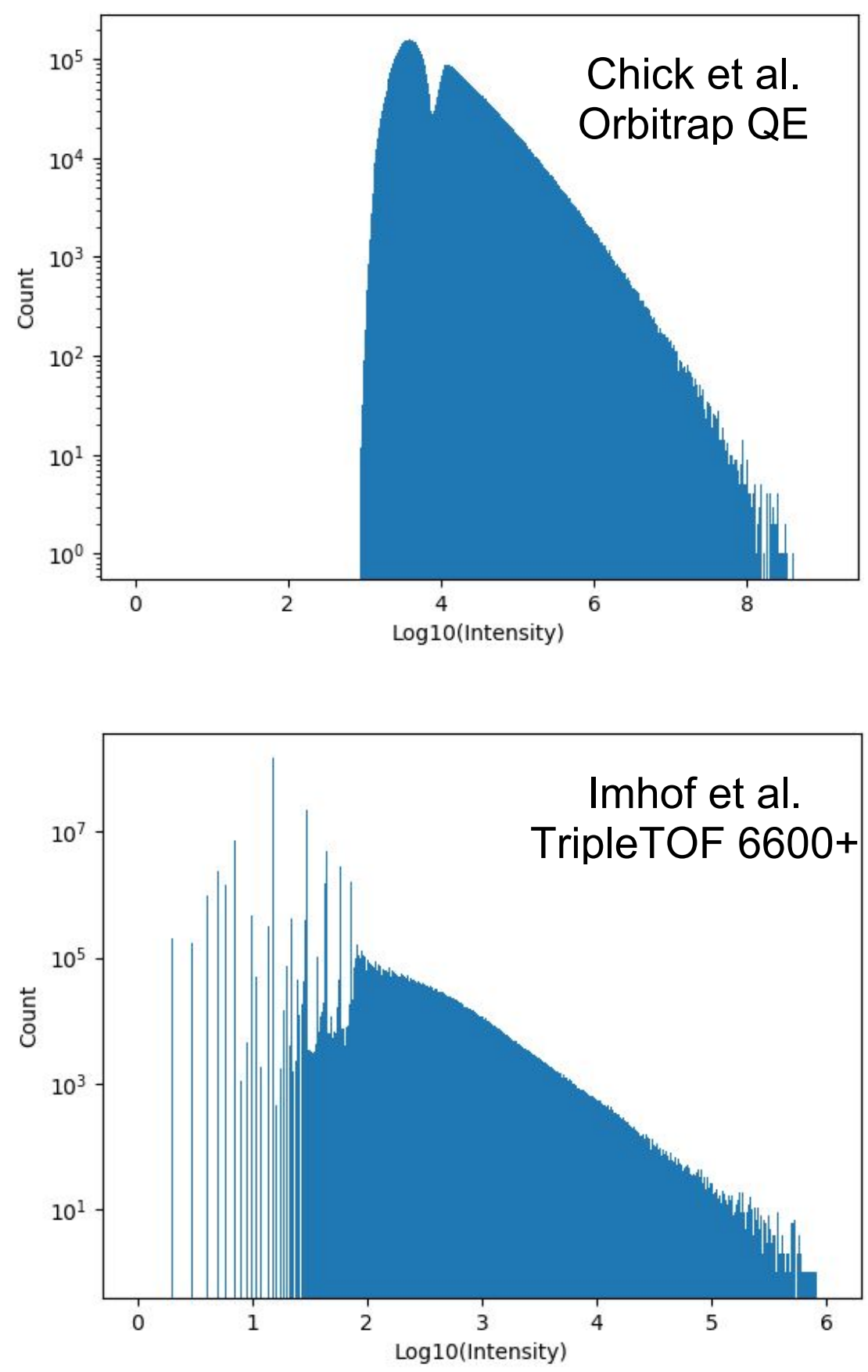

Figure S4. Distribution of MS2 peak intensities from Chick and Ammar et al. datasets. Both axes are $\log (10)$ transformed. Data from all scans in a raw file has been combined into a single plot. Ammar data shows numerous peaks at low and discontinuous intensity values that correspond to noise. 


\section{References}

1. Chick, J. M.; Kolippakkam, D.; Nusinow, D. P.; Zhai, B.; Rad, R.; Huttlin, E. L.; Gygi, S. P., A mass-tolerant database search identifies a large proportion of unassigned spectra in shotgun proteomics as modified peptides. Nature Biotechnology 2015, 33 (7), 743-749.

2. Riley, N. M.; Hebert, A. S.; Westphall, M. S.; Coon, J. J., Capturing site-specific heterogeneity with large-scale N-glycoproteome analysis. Nature Communications 2019, 10 (1), 1311-1311.

3. $\quad$ Anapindi, K. D. B.; Yang, N.; Romanova, E. V.; Rubakhin, S. S.; Tipton, A.; Dripps, I.; Sheets, Z.; Sweedler, J. V.; Pradhan, A. A., PACAP and other neuropeptide targets link chronic migraine and opioidinduced hyperalgesia in mouse models. Molecular and Cellular Proteomics 2019, 18 (12), 2447-2458.

4. Espadas, G.; Borràs, E.; Chiva, C.; Sabidó, E., Evaluation of different peptide fragmentation types and mass analyzers in data-dependent methods using an Orbitrap Fusion Lumos Tribrid mass spectrometer. Proteomics 2017, 17 (9), 1600416.

5. Meier, F.; Brunner, A.-D.; Koch, S.; Koch, H.; Lubeck, M.; Krause, M.; Goedecke, N.; Decker, J.; Kosinski, T.; Park, M. A.; Bache, N.; Hoerning, O.; Cox, J.; Räther, O.; Mann, M., Online Parallel Accumulation-Serial Fragmentation (PASEF) with a Novel Trapped Ion Mobility Mass Spectrometer. Molecular \& cellular proteomics : MCP 2018, 17 (12), 2534-2545.

6. Ammar, C.; Berchtold, E.; Csaba, G.; Schmidt, A.; Imhof, A.; Zimmer, R., Multi-Reference Spectral Library Yields Almost Complete Coverage of Heterogeneous LC-MS/MS Data Sets. Journal of Proteome Research 2019, 18 (4), 1553-1566.

7. Hoopmann, M. R.; Finney, G. L.; MacCoss, M. J., High-speed data reduction, feature detection, and MS/MS spectrum quality assessment of shotgun proteomics data sets using high-resolution mass spectrometry. Analytical Chemistry 2007, 79 (15), 5620-5632.

8. Hoopmann, M. R.; MacCoss, M. J.; Moritz, R. L., Identification of peptide features in precursor spectra using hardklör and krönik. Current Protocols in Bioinformatics 2012, (SUPPL.37), 1-13.

9. Tay, A. P.; Liang, A.; Hamey, J. J.; Hart-Smith, G.; Wilkins, M. R., MS2-Deisotoper: A Tool for Deisotoping High-Resolution MS/MS Spectra in Normal and Heavy Isotope-Labelled Samples. Proteomics 2019, 19 (17), 1800444-1800444.

10. Breen, E. J.; Hopwood, F. G.; Williams, K. L.; Wilkins, M. R., Automatic Poisson peak harvesting for high throughput protein identification. Electrophoresis 2000, 21 (11), 2243-2251.

11. French, W. R.; Zimmerman, L. J.; Schilling, B.; Gibson, B. W.; Miller, C. A.; Townsend, R. R.; Sherrod, S. D.; Goodwin, C. R.; McLean, J. A.; Tabb, D. L., Wavelet-based peak detection and a new charge inference procedure for MS/MS implemented in proteo wizard's msconvert. Journal of Proteome Research 2015, 14 (2), 1299-1307. 